\title{
Sighting from the cyclopean eye: The cyclops effect in preschool children
}

\author{
RAPHAEL BARBEITO \\ University of Waterloo, Waterloo, Ontario, Canada
}

\begin{abstract}
The behavior of preschoolers in two sighting tasks was observed. In one task, the child was asked to sight a target through a tube, and, in the other, to point to a target. In addition to monocular sighting, the "cyclops effect" was observed; the child positioned the tube or finger in line with the target and a point between the eyes and left it there. Observation of both types of response suggests a developmental shift from central sighting, which reflects the visual direction basis of sighting tasks, to monocular sighting, which reflects the monocular alignment required by the tasks.
\end{abstract}

Church $(1966,1970)$ anecdotally reported that when $1 \frac{1 / 2}{2}$ to 2 -year-olds were encouraged to sight through a tube, they did so by placing the tube squarely between the two eyes, rather than over one eye. This "cyclops" effects, as Church called it, seems counterintuitive for at least two reasons. First, from our adult perspective, we know that to look at a stimulus through a tube we must place it over one eye as, for example, when looking through a telescope. Second, the cyclops effect is inconsistent with what may be expected given the widespread use of a variety of analogous sighting tasks to determine ocular dominance. Sighting tasks essentially require the alignment of two stimuli, and it is well documented that school-aged children and adults consistently position the stimuli collinear with one particular eye, a behavior often used to operationally define ocular dominance (see, for example, Porac \& Coren, 1976).

Two possible explanations for the cyclops effect seem possible. First, such responses may simply be a reflection of random variability. Data which show that preschool children are less reliable than adults in the preference for one eye in sighting tests suggest this possibility (e.g., Updegraff, 1932).

A second possibility is that the effect is, in fact, nonrandom variability which reflects a real bias to look or "sight" from a point between the eyes. Consistent with the latter view are data which suggest that behavior in sighting tasks is related to the processing

The author wishes to thank H. Ono, J. Corfield, and M. P. Bryden for their valuable comments on an earlier version of this paper, and to especially thank S. M. Tapley for testing the children. The cooperation of the Early.Childhood Education Centre of the University of Waterloo is acknowledged. The author was supported by a postdoctoral fellowship from the Natural Sciences and Engineering Research Council of Canada. The author's present address: School of Optometry, University of Houston, Central Campus, Houston, Texas 77004. of visual direction specified from a visual direction center located between the eyes (Barbeito, 1981; Charnwood, 1949) and recent data which clearly show that the center is between the eyes, not in the dominant eye (Ono \& Barbeito, 1982). Another element that supports this possibility is that when stimuli are objectively aligned with either eye, they appear to be aligned to a point between the eyes. For example, when a tube is placed over one eye and the far end fixated, the tube appears to be aligned to the nose (Roelofs, 1959). Analogous results were reported by Ono and Barbeito (1982) for the stimuli in a sighting task. They asked subjects to indicate the apparent location of the stimuli after the subjects had adjusted them to be aligned to one eye, as per the usual procedure of the task. As suggested by the Roelofs' tube example, they found that the objective and apparent location of the stimuli in the sighting task did not coincide; the stimuli were perceived as being collinear with a point between the eyes as opposed to being seen collinear with the eye to which they were objectively aligned. It is as if the stimuli were seen from a point between the eyes. (For a more complete discussion of the discrepancy between objective and subjective localization, and other demonstrations of it, see Howard, 1982, p. $283 \mathrm{ff}$, Howard \& Templeton, 1966, p. 274ff, or Ono, 1979).

Perceiving stimuli from a point located between the two eyes implies a conflict with the objective alignment of stimuli to one eye required by sighting tasks. Reports of adults' behavior in sighting tasks which show that adults align stimuli with a point between the eyes en route to achieving the monocular alignment (Barbeito, 1980, 1981; Covell, 1951) suggest that this conflict is still evident in adults, but that they have developed strategies to resolve it. The cyclops effect, that is, central alignment without the final correction to monocular alignment when sighting, may be an indication that children have not yet developed such strategies. 
Unfortunately, while Church (1966) states that the cyclops effect persists well into the preschool years, his report is anecdotal and he provides no data. Thus, the status of this phenomenon cannot be determined from his report. The purpose of the report described here, then, is to evaluate sighting behavior in preschoolers with the specific aim of determining the frequency of occurrence of the cyclops effect, and to evaluate whether such responses are attributable to random error. To this end, two sighting tasks were used. In one task, children were asked to sight a target through a tube, the task reported by Church (1966). In the other, they were asked to point to the target. The second task was used to evaluate the generalizability of the phenomenon.

\section{METHOD}

The child entered the experimental room with the tester and was seated on one side of a square table. The tester sat facing the child across the table, approximately $1.5 \mathrm{~m}$ away. Testing was then begun. The target to be sighted was the nose of the tester (after Porac \& Coren, 1975). For the pointing task, the child was told, "I want you to point to my nose." After pointing, the tester asked the child to lower his/her arm, if necessary. For the tube task the child was shown a $20-\mathrm{cm}$-long $\times 2.5-\mathrm{cm}$-diam tube and told, "Now, I want you to look at my nose through the tube." The tube was already lying on the table in front of the child, who was also instructed to use both hands when holding it. Again, if necessary, after sighting with the tube the child was told to replace the tube on the table.

Certain restrictions were placed on the child's behavior when responding. In the pointing task, the child was to point with a straight, index finger and with arm extended. Also, the finger was to be in the plane defined by the child's eyes and the target. Similarly, for the tube task, the tube was to be held near the face in this plane and brought to that position directly from the table top. Furthermore, both eyes were to be kept open during the entire experiment. It was not necessary to inform the child of these restrictions via instructions as most children conformed to them without being told. Thus, additional instructions were used only as the situation warranted.

All children began the experiment with one trial of the pointing task.' As the experiment continued, the trials alternated between the two tasks. This procedure was used to achieve and maintain the child's interest in the experiment. Each child had four trials in each task. The entire testing session took less than 5 min per child.

The response in each of the four trials of both tasks was judged and recorded by the tester as monocular, central, or unscorable. In the tube task, the monocular and central "scoring" possibilities reflected the place on the child's face that was covered by the tube, given that it was directed at the target. For example, if the tube was placed in such a position that the child could view the target through the tube, the response was recorded as a monocular response. If the tube was positioned at eye level but between the two eyes so that neither eye could view the target through the tube, the response was recorded as central. A response assigned to this category was considered an observation of the cyclops effect. Because of the tube's diameter relative to the interocular distance of the children, a central response is tantamount to the tube's being placed squarely between the eyes, that is, over the bridge of the nose.

For the pointing task, an analogous "scoring" procedure was used; the tester was required to judge the place on the child's face that was in line with the target and the end of the child's finger. If the finger was not in line with either eye but with a point between the two eyes, the response was assigned to the cen- tral category. To reduce errors in judgment produced by the tester's perspective, and to establish the criterion for each response type, the author and tester rehearsed the "scoring" techniques in a pilot experiment. For both tasks, the judgment was based on the end point of the response. That is, if the finger/tube was first aligned centrally and then shifted over to be aligned with one eye, that response was categorized as monocular.

Responses placed in the unscorable category were those that did not fit the monocular or central classifications, usually because they did not adhere to the strict behavioral requirements. These responses may be considered indicative of variable error. Examples of unscorable responses include placement of the tube over one eye or between the eyes but not directed at the target and alignment of the finger and target to a point on the temporal side of an eye.

The female tester was very experienced at administering sighting tasks and scoring the responses as either right- or left-eye sighting, that is, as monocular, but she was otherwise naive as to the purpose of the study. Before the experiment began, the tester went through a familiarization period with the children in their normal preschool environment.

The subjects were 34 children from a preschool. The criteria for selection were the parents' permission, the child's willingness to participate, and a sufficient command of English on the part of the child to understand the instructions. At the time of testing, the children ranged in age from 3 years 5 months to 5 years 5 months. ${ }^{2}$

\section{RESULTS}

To determine the extent to which preschoolers exhibit the cyclops effect, the frequency of responses judged to be monocular or central in each task was calculated. These responses totaled 90 in the tube task and 98 in the pointing task. The data, expressed as percentages of the sum of these two types of response, are presented in Table 1 with the children divided into two groups of equal size to reflect possible age differences. The younger group included children ranging in age from 3 years 5 months to 4 years 2 months, the older group from 4 years 4 months to 5 years 5 months. ${ }^{3}$ While an attempt was made to keep the child squarely facing the tester, this was not always achieved. On some trials in the tube task, the child rotated his/her head to such an extent that only one eye could see the tester. Since head turning predisposes the child to make a monocular response, data from such trials were excluded from this analysis.

The cyclops effect was observed in both the tube and pointing tasks. Furthermore, it was observed

Table 1

Percent Monocular and Central Responses in Each Sighting Task

\begin{tabular}{lcc}
\hline & \multicolumn{2}{c}{ Age Group } \\
\cline { 2 - 3 } Response & Younger & Older \\
\hline Tube Task & \\
Central & 66.0 & 88.4 \\
& 34.0 & 11.6 \\
Monocular & Pointing Task & 81.8 \\
Central & 63.5 & 18.2 \\
\hline
\end{tabular}


more frequently in the younger group for both tasks. The occurrence of the effect is particularly interesting in the tube task. Because adults know that to sight a target through a tube it must be placed over one eye, responses categorized as central may not have been expected. Yet, in more than a third of the cleanly "scored" trials (those categorized as either monocular or central), children in the younger group placed the tube squarely between the eyes, and kept it there! Thus, the anecdotal report of Church (1966) is empirically verified.

To evaluate the possible age-relatedness of the cyclops effect, the number of children in each age group who gave at least one central response and the number of those who gave none were determined for each task and the frequencies were compared using the chi-square statistic. In the tube task, the frequencies were 6 central and 5 no-central for the younger group, and 0 central and 10 no-central for the older group. The $\chi^{2}=5.20, \mathrm{p}<.025$ (using Yates's correction), and indicates a relationship between age and the cyclops effect. The frequencies for the pointing task were 10 central and 6 no-central for the younger group, and 6 central and 11 no-central for the older group. (One child was excluded because she gave no scorable responses in this task.) While these frequencies yield a nonsignificant $\chi^{2}=2.44, \mathrm{p}>.05$, they are in a direction consistent with that indicated by the tube task data.

To further evaluate the age-relatedness of the effect, the number of children who showed it at least once in either task was determined for each age group. In the younger group, 14 of 17 children showed the effect; in the older group, 6 of 17 did. These proportions yield a significant chi-square value $\left(\chi^{2}=7.77, \mathrm{p}<.01\right)$, which clearly indicates an association between age and observation of the cyclops effect. ${ }^{4}$

Is the cyclops effect the result of a variable error? To consider this possibility, the unscorable trials were evaluated. Of primary interest are the data of the tube task because it is the one described by Church (1966). Of the 136 trials conducted, only 5 were unscorable, and no child who evidenced the cyclops effect gave an unscorable response. Furthermore, if the cyclops effect simply reflected random error, there is no a priori reason for the error to be restricted to the midline side of the eye. Thus, placement of the tube on the temporal side of the eye should have been observed. No such responses were observed.

The frequency of unscorable trials was greater in the pointing task, totaling 30 of the 136 trials. The two most frequent types of unscorable trials were the finger's not being held sufficiently still for its alignment to be judged and the finger's being in line with a point on the temporal side of an eye. While this error rate is higher than that found in the tube task, those children responsible for the higher error rate ac- counted for fewer than one-quarter of the central responses -6 of 27 . Furthermore, 12 of the 17 children who exhibited the cyclops effect in this task gave no unscorable responses. It seems unlikely, therefore, that the bulk of responses scored as central can be attributed to random variability in pointing.

\section{DISCUSSION}

The data presented for the tube task confirm Church's (1966) anecdotal report of the cyclops effect in young children and, as such, provide the first empirical documentation of this phenomenon. The results of the pointing task show that the effect is generalizable across sighting tasks. The data show clearly that the cyclops effect is not attributable simply to the fact that children of this age are not as consistent as adults in the choice of which of the two eyes is used for sighting. Rather, the effect represents something qualitatively different from the monocular alignment normally reported in sighting tasks; it represents a real bias to "sight" from a point between the eyes.

Earlier formal studies of young children's sighting behavior (e.g., Coren, Porac, \& Duncan, 1981; Scheidemann \& Robinette, 1932; Updegraff, 1932) may have failed to report the cyclops effect because such responses were probably considered to be errors within the context of the use of these tasks as operational definitions of ocular dominance. As research on ocular dominance began to peak, Dolman (1919) devised a sighting task to determine the dominant eye. The task was to sight a target through an aperture in a card. The advantage of his sighting task, he argued, was that it eliminated "a certain percentage of negative results which are obtained when the first and second test objects are aligned with some point between the two eyes' (Dolman, 1919, p. 867). Indeed, if a researcher is using a sighting task simply as a measure of left or right "eyedness," it is easy to understand why central alignment would be considered a nuisance. This attitude could explain why studies dealing with sighting in preschool children would not have reported the cyclops effect, even though two such studies (Scheidemann \& Robinette, 1932; Updegraff, 1932) reported difficulty in obtaining measures of ocular dominance.

In what context can the cyclops effect be understood? Several reports suggest that adult sighting behavior bears some resemblance to the cyclops effect observed here. Helmholtz (1910/1925, p. 259) noted long ago that, when pointing to a target, errors are made toward the midline. This "error" was investigated in adults by Barbeito $(1980,1981)$, who administered the pointing task under two conditions-a closed-loop condition, in which the usual procedure of the task was followed, and an open-loop condition, in which the subject was not permitted feedback concerning the position of the finger relative to the target. In the closed-loop condition, 47 of the 48 
adults tested placed the finger in line with one eye and the target, but only after first placing the finger in line with the target and a point between the eyes. This behavioral pattern was also observed in the other sighting task he investigated-the card test, which requires the target to be sighted through a small aperture in the center of a card. When these two sighting tasks were modified to remove the constraint of monocular alignment, adults did not align the stimuli to one eye but aligned them to a point between the eyes. This latter observation was also reported by Charnwood (1949) and Murroughs and Christakos (1949) for another sighting task. Thus, with specific modifications to sighting tasks, adults, too, exhibit the cyclops effect, and, even under the usual procedures of the tasks, adults appear to show vestiges of the effect.

Why a point between the eyes? Recently, Ono and Barbeito (1982) evaluated the hypothesis that visual directions are judged from the eye preferred for sighting, that is, the sighting dominant eye, as opposed to the cyclopean eye, a theoretical point centrally located between the eyes. They clearly showed that the stimuli in a sighting task are not perceived from the dominant eye, but from the cyclopean eye. They suggest that the hypothesis that the dominant eye is the center of visual direction arises from the expectancy that because we see with our eyes, we also see from our eyes.

The subjective reality of perceiving from the cyclopean eye and the objective reality of seeing with our eyes are brought into conflict in sighting tasks. The notion that we see from the cyclopean eye explains various aspects of adult sighting behavior discussed above, for example, why adults align stimuli in a sighting task centrally before aligning them monocularly. If children, too, see from the cyclopean eye, perhaps they also expect to be able to see with it, and so try to sight with that point. The fact that adults do not exhibit the cyclops effect and preschool-aged children do, implies that resolution of the conflict is subject to developmental processes. The observation made in the present study that both types of response occur in the same child suggests that children of preschool age are at some intermediate stage in the resolution.

\section{REFERENCES}

Barbe ITO, R. Ocular dominance: An explanation based on sighting behaviour. Doctoral dissertation, York University, Toronto, 1980.

BARBeito, R. Sighting dominance: An explanation based on the processing of visual direction in tests of sighting dominance. Vision Research, 1981, 21, 855-860.

Barbeito, R., \& Ono, H. Four methods of locating the egocenter: A comparison of their predictive validities and reliabilities. Behavior Research Methods \& Instrumentation, 1979, 11, 31-36.

Charnwoon, J. R. B. Observations on ocular dominance. Optician, 1949, 118, 85-88, 96.
Сhurch, J. Language and the discovery of reality. New York: Vintage, 1966.

Church, J. Techniques for the differential study of cognition in early childhood. In J. Hellmuth (Ed.), Cognitive studies (Vol. 1). New York: Brunner, 1970.

Conen, S., Porac, C., \& Duncan, P. Lateral preference behaviors in preschool children and young adults. Child Development, 1981, 52, 443-450.

Covell, M. Relation of eye dominancy test to other visual data. Optometric Weekly, 1951, 42, 1297-1299.

Dolman, P. Tests for determining the sighting eye. American Journal of Ophthalmology, 1919, $2,867$.

Helmholtz, H., von [Handbook of physiological optics] (Vol. 3) (P. C. Southall, Ed. and trans.). New York: Dover, 1925. (Originally published, 1910.)

Howard, I. P. Human visual orientation. Chichester, England: Wiley, 1982.

Howard, I. P., \& Templeton, W. B. Human spatial orientation. New York: Wiley, 1966.

Murnoughs, T. R., \& Christakos, J. Ambiguity in ocular dominance. Optician, 1949, 118, 449-451.

Ono, H. Axiomatic summary and deductions from Hering's principles of visual direction. Perception \& Psychophysics, $1979,25,473-477$.

Ono, H., \& Barbeito, R. The cyclopean eye vs. the sightingdominant eye as the center of visual direction. Perception \& Psychophysics, 1982, 32, 201-210.

Porac, C., \& Conen, S. Is eye dominance a part of generalized laterality? Perceptual and Motor Skills, 1975, 40, 763-769.

Porac, C., \& Coren, S. The dominant eye. Psychological Bulletin, 1976, 83, 880-897.

Roelofs, C. O. Considerations on the visual egocentre. Acta Psychologica, 1959, 16, 226-234.

Scheidemann, N. V., \& Robinette, G. E. Testing the ocular dominance of infants. Psychological Clinic, 1932, 31, 62-63.

UPDEGRAFF, R. Ocular dominance in young children. Journal of Experimental Psychology, 1932, 15, 758-766.

\section{NOTES}

1. For the most part, the preferred hand was used. Initially, an attempt was made to vary the hand used, with the intention of evaluating the effects of this manipulation. Children had difficulty pointing with the nonpreferred hand, so the procedure was dropped.

2. Visual functions of the children were not evaluated. Thus, it is possible that some children without normal binocular vision were tested. This would decrease the possibility of observing the cyclops effect.

3. There were responses for which the tester could not decide between the central and monocular categorizations, as when the tube was placed partly over the bridge of the nose and partly over the nasal portion of one eye. It is quite possible that such responses reflect the cyclops effect because the cyclopean eye is often located off the midline, nearer one eye (Barbeito, 1981; Barbeito \& Ono, 1979; Ono \& Barbeito, 1982). However, because it could not be definitely stated that the child could not actually have been seeing the target through the tube or that the finger and eye were not actually aligned in such responses, these trials were excluded from all analyses. These trials totaled 13 of the tube task -7 in the younger group, 6 in the older-and 8 in the pointing task- 5 in the younger group and 3 in the older.

4. Those children for whom the head-turning problem could not be controlled were excluded from this analysis. Ignoring the problem altogether and counting these children gave frequencies of 8-8 in the younger group and 3-14 in the older group. These frequencies yield a $\chi^{2}=3.88, p<.05$. (One child had an incomplete set of data for this task and was not counted.)

(Manuscript received September 27, 1982; revision accepted for publication February 9, 1983,) 